EXTENDED REPORT

\title{
Effects of combined cataract surgery and trabeculectomy with mitomycin $C$ on ocular dimensions
}

\author{
S K Law, A M Mansury, D Vasudev, J Caprioli
}

Br J Ophthalmol 2005;89:1021-1025. doi: 10.1136/bjo.2004.060053

See end of article for authors' affiliations

\section{Correspondence to:}

Simon K Law, MD, 100

Stein Plaza \#2-235, Jules

Stein Eye Institute, Los

Angeles, CA 90095, USA;

law@jsei.ucla.edu

Accepted for publication

2 December 2004

\begin{abstract}
Aims: To characterise changes in ocular dimensions after combined cataract operation and trabeculectomy with mitomycin C using separate incisions (combined operation).

Methods: 24 consecutive eyes that had combined operation and 16 eyes that had cataract operation alone were enrolled. The axial lengths before and after operations were determined with non-contact optical coherence biometry. The intraocular pressures (IOP), axial lengths, corneal curvatures, and the expected and observed refractive errors before and after operations were compared.

Results: After a combined operation, mean IOP was significantly reduced from 16.6 (SD 5.8$) \mathrm{mm} \mathrm{Hg}$ to $10.9(4.1) \mathrm{mm} \mathrm{Hg}(p<0.00001)$, and mean axial length was significantly shortened from 24.10 (0.98) $\mathrm{mm}$ to $23.98(0.96) \mathrm{mm}(\mathrm{p}<0.00001)$. The mean axial length reduction after combined operation (117 $(57) \mu \mathrm{m}$ ) was significantly larger than the reduction after cataract operation alone (75 (38) $\mu \mathrm{m}, \mathrm{p}<0.02)$, and correlated significantly with the postoperative IOP $(p<0.002)$. There was a mean with the rule surgically induced corneal astigmatism of $0.44(0.83)$ dioptre by vector analysis, and a significant increase of mean keratometry reading of $0.23(0.46)$ dioptre after a combined operation. However, there was no significant difference between the expected and observed refractive errors.

Conclusions: Despite an alteration of the axial length and corneal curvature, the refractive outcome after a combined operation did not differ significantly from the predicted refraction.
\end{abstract}

C ombined cataract operation and trabeculectomy is occasionally required for visual rehabilitation and prevention of progressive optic nerve damage, in patients with coexisting cataract and glaucoma. Studies on patients with trabeculectomy have shown a significant decrease of axial length (AL) after surgery. ${ }^{1-3}$ An alteration of the AL from the preoperative measurement may affect the intraocular lens (IOL) calculation and ultimately the postoperative refraction in patients undergoing a combined operation. Previous reports have also shown that there is induced corneal astigmatism after trabeculectomy and combined operation with various surgical techniques. ${ }^{3-9}$ According to an evidence based review of the surgical techniques and adjuvant used in combined operation, the strongest evidence of efficacy exists for using mitomycin C, separating the incisions for cataract and glaucoma surgery, and removing the nucleus by phacoemulsification. ${ }^{10}$ It is unclear whether combined operation with this technique will induce a change of $\mathrm{AL}$ and corneal astigmatism, and affect the postoperative refraction.

Previous reports on changes of AL after trabeculectomy were based on ultrasound biometry, which requires physical contact with the eye by a transducer or a saline immersion bath. ${ }^{1-3}$ Recently, a non-contact, optical coherence biometry method based on the principle of partial coherence interferometry has received wide acceptance in providing accurate calculation of IOL power for cataract surgery. ${ }^{11}{ }^{12}$ This method may allow accurate measurement of the AL even when the eye is softened after glaucoma surgery without indentation or deformation of the eye as in contact ultrasound biometry.

The purpose of this study was to characterise the changes of ocular dimensions including the AL and corneal curvature, and the expected and observed refractive errors, in uncomplicated combined phacoemulsification cataract operation with IOL implantation and trabeculectomy with mitomycin C application, using separate incisions (combined operation).

\section{PATIENTS AND METHODS}

This study was approved by the institutional board of the University of California, Los Angeles. The optical coherence biometer, IOLMaster V.2.02 (Zeiss-Humphrey, Dublin, CA, USA), was available as one of the methods to measure the AL for IOL power calculation in our practice since February 2002. The optical coherence biometer measures the AL based on the principle of partial coherence interferometry without touching the eye. The medical records of patients who had combined operation between February 2002 and April 2004 (26 months), and patients who had cataract operation alone between August 2003 and April 2004 (8 months), without previous intraocular operation by one glaucoma specialist (SKL) were reviewed. A shorter enrolment period was needed for cataract operation because more patients received cataract operation alone than combined operation in our practice. Eyes in which accurate AL measurements with the IOLMaster were unobtainable were excluded, either because there was not enough light reflected back from the eye with advanced cataract, or the patient was unable to fixate during the measuring period. Patients who had the AL measurement by the non-contact optical coherence biometry were contacted to return for a follow up visit with the intraocular pressure (IOP), AL, corneal curvature, and refraction measured. If both eyes of a patient were qualified, only the first eye that had theoperation was enrolled (table 1).

The preoperative IOP was recorded from the office visit closest to the date of the preoperative $\mathrm{AL}$ and corneal curvature measurement. There was no change in glaucoma management during the interval. Postoperative measurements of the IOP, AL, automated keratometry, and postoperative refraction were obtained on the same day.

An experienced operator performed the AL measurements with the IOLMaster. Evaluation of the AL graph by the

Abbreviations: $A L$, axial length; $A T R$, against the rule; $I O L$, intraocular lens; IOP, intraocular pressure; WTR, with the rule 
Table 1 Reasons for exclusion for combined operation group and cataract operation group

\begin{tabular}{|c|c|c|}
\hline & $\begin{array}{l}\text { Combined } \\
\text { operation group }\end{array}$ & $\begin{array}{l}\text { Cataract } \\
\text { operation group }\end{array}$ \\
\hline Enrolment period (no of months) & $\begin{array}{l}\text { Feb } 2003 \text { to Apr } 2004 \\
\text { ( } 26 \text { months) }\end{array}$ & Jul 2003 to Apr 2004 (9 months) \\
\hline $\begin{array}{l}\text { No of prospective eyes } \\
\text { Reasons of exclusion: }\end{array}$ & 50 eyes (50 patients) & 36 eyes (36 patients) \\
\hline $\begin{array}{l}\text { IOLMaster measurement unobtainable } \\
\text { Lost to follow up } \\
\text { No of enrolled eyes }\end{array}$ & $\begin{array}{l}23 \text { eyes } \\
3 \text { eyes } \\
24 \text { eyes ( } 24 \text { patients) }\end{array}$ & $\begin{array}{l}8 \text { eyes } \\
12 \text { eyes } \\
16 \text { eyes ( } 16 \text { patients) }\end{array}$ \\
\hline
\end{tabular}

operator was required on all patients to ensure proper $\mathrm{AL}$ determination. Five measurements were obtained for each eye through an undilated pupil, with no anaesthesia, with the patient in a sitting position, and an average was calculated. "Phakic" and "Pseudophakic Acryl" settings of the IOLMaster were used to determine the preoperative and postoperative AL respectively. The IOLMaster also obtained three automated keratometry measurements for each eye and an average was calculated. Postoperative refraction was obtained using an automated refractor (Nikon Auto Refractokeratometer Speedy-K, Nikon Instruments Inc, Torrance, CA, USA), followed by manifested refraction. Three IOL calculation formulas (SRK/T, Holladay, and Hoffer Q) were used to determine the IOL power for all cases. An average IOL power obtained from the results of the three formulas was obtained. The one piece foldable acrylic IOL (model SA60AT by Alcon Laboratory, Fort Worth, TX, USA) was used for all cases.

The combined operation was performed with separated incision sites with the cataract surgery performed first. Bimanual phacoemulsification of the nucleus and aspiration of the cortex was performed through a $3 \mathrm{~mm}$ temporal clear cornea incision created with a diamond keratome. An IOL was placed in the capsular bag. The corneal incision was closed with a single interrupted 10-0 nylon suture. No corneal suture was required in cataract operation alone.

For the trabeculectomy, a superior limbal based conjunctival flap was created. A $4.0 \mathrm{~mm} \times 3.0 \mathrm{~mm}$ scleral flap was outlined with a surgical blade at the 12 o'clock position. A piece of cut Weck-cel sponge (approximately $6.0 \mathrm{~mm} \times$ $3.0 \mathrm{~mm})$ soaked in mitomycin C $(0.3 \mathrm{mg} / \mathrm{ml})$ was placed under the Tenon's capsule and conjunctiva on the sclera for 1 minute. The Weck-cel piece was removed followed by thorough irrigation of the area exposed to mitomycin $\mathrm{C}$ with $30 \mathrm{ml}$ balanced salt solution. A partial thickness sclera flap was dissected. A block of trabecular section was removed anterior to the scleral spur with a Kelly punch, and a peripheral iridectomy was performed. The scleral flap was sutured at its two corners with interrupted 10-0 nylon sutures to ensure a slight egress of aqueous, yet maintain a deep anterior chamber. Finally, the conjunctiva and Tenon's capsule were sutured with a continuous 9-0 polyglactin suture (Vicryl).

Suture lysis was performed with the argon laser for inadequate IOP control or a low filtering bleb between 1-4 weeks after the combined operation. The target IOP before laser suture lysis or the addition of ocular hypotensive therapy was generally the low teens, with the goal of preventing further progression of functional visual loss. The clear corneal suture was removed only if the suture induced high astigmatism of $>3$ dioptres and visual quality might be improved by its removal.

The surgically induced astigmatism was calculated using the method described by Cravy. ${ }^{13}$ This method provides a mathematical expression of the change in astigmatism with the rule (WTR) or against the rule (ATR). A change that increased the curvature of the vertical meridian was defined as WTR change and given a plus designation, and one that decreased curvature was defined as ATR change and given a minus designation.

Statistical analysis was performed with SPSS for Windows (SPSS, Chicago, IL, USA). Paired two sample $t$ tests were performed to compare the preoperative and postoperative values of IOP, axial length, mean corneal curvature, and the expected and observed refractive errors; and non-paired two sample $t$ tests were performed to compare between the combined operation and cataract operation groups. The relation between axial length and IOP was analysed with regression analysis. A two tailed p value less than 0.05 was considered statistically significant.

\section{RESULTS}

A total of 24 eyes (24 patients) with combined operation and 16 eyes (16 patients) with cataract operation alone were included in the study (table 1). The demographic data were represented in table 2 . The mean interval between the recorded preoperative IOP and the preoperative AL and

Table 2 Demographic data of patients with combined operation and cataract operation

\begin{tabular}{llll}
\hline & $\begin{array}{l}\text { Combined } \\
\text { operation group } \\
\text { 24 eyes (24 patients) }\end{array}$ & $\begin{array}{l}\text { Cataract } \\
\text { operation group } \\
16 \text { eyes (16 patients) }\end{array}$ & p Value \\
\hline $\begin{array}{l}\text { Mean age (years (SD)) } \\
\text { Sex }\end{array}$ & $74.8(11.7)$ & $75.9(7.2)$ & 0.82 \\
$\quad$ Female/male & $10 / 14$ & $9 / 7$ & 0.35 \\
$\begin{array}{l}\text { Race } \\
\quad \text { White }\end{array}$ & 14 & 10 & 0.52 \\
$\quad$ Non-white & 10 & 6 & $<0.0001$ \\
$\begin{array}{l}\text { Diagnosis } \\
\quad \text { Glacoma or glaucoma } \\
\text { suspect }\end{array}$ & 24 & 11 & \\
$\quad$ Without glaucoma & 0 & 5 & \\
\hline
\end{tabular}




\begin{tabular}{|c|c|c|c|c|}
\hline & Preoperative & Postoperative & $\begin{array}{l}\text { Difference between } \\
\text { preoperative and } \\
\text { postoperative } \\
\text { values }\end{array}$ & $\mathrm{p}$ Value \\
\hline Mean IOP (mm Hg) (SD) & $16.6(5.8)$ & $10.9(4.1)$ & $-6.0(4.9)$ & $<0.00001$ \\
\hline Mean axial length (mm) (SD) & $24.10(0.94)$ & $23.98(0.96)$ & $-0.12(0.06)$ & $<0.00001$ \\
\hline $\begin{array}{l}\text { Mean keratometry (dioptres) } \\
\text { (SD) }\end{array}$ & $43.76(1.15)$ & $44.00(1.24)$ & $0.23(0.46)$ & 0.02 \\
\hline $\begin{array}{l}\text { Mean refractive error } \\
\text { (spherical equivalent in } \\
\text { dioptres) (SD) }\end{array}$ & $-0.23(0.12)$ & $-0.01(0.90)$ & $0.22(0.91)$ & 0.25 \\
\hline
\end{tabular}

corneal curvature measurements was 24.3 (SD 28.1) days. No change of glaucoma management occurred during this interval. The mean interval between the surgery and the postoperative AL and corneal curvature measurements was 7.3 (6.0) months. The 10-0 nylon corneal suture used in the combined operation was removed in one eye at 40 days after surgery because of the high astigmatism induced. Another eye had a loosened corneal suture that required removal at 8.6 months after the surgery. Sixteen $(66.7 \%)$ of the 24 eyes that had combined operations had laser suture lysis performed to adjust the postoperative IOP. Mean interval of suture lysis was 8.4 (8.8) days postoperatively. Twelve (75\%) of the 16 eyes had two (all) sutures lysed, and four eyes (25\%) had one of the sutures lysed with laser.

Tables 3 and 4 summarised the comparison of the preoperative and postoperative IOP, AL, corneal curvature, and refractive error in eyes that had combined operations and cataract operations, respectively. Table 5 summarised the comparison of changes of measurements between the two groups. The postoperative AL was significantly reduced after both operations, with the mean AL reduction after the combined operation (117 (57) $\mu \mathrm{m}$, range 30-220 $\mu \mathrm{m})$ significantly larger than the mean reduction after the cataract operation (75 (38) $\mu \mathrm{m}$, range $20-120 \mu \mathrm{m}, \mathrm{p}<0.02$ ). There was a significant difference between the preoperative and postoperative mean keratometry readings in the combined operation group, but not the cataract operation group. The AL reduction from combined operation correlated significantly with the postoperative IOP $\left(p<0.002, R^{2}=0.408\right)$, but no significant correlation between the AL reduction and the reduction of IOP $\left(p=0.45, R^{2}=0.028\right)$ (figs 1,2$)$. The $A L$ reduction from cataract operation was not significantly correlated with the preoperative, postoperative, or change of IOP. Figure 3 represented the correlation between AL reduction and postoperative IOP after cataract operation

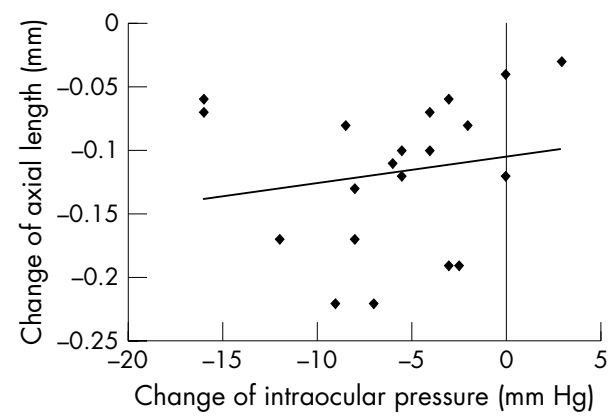

Figure 1 Correlation between change of axial length and change of intraocular pressure from combined operation $(p=0.454, y=0.002 \times$ $-0.105, R^{2}=0.030$ ).

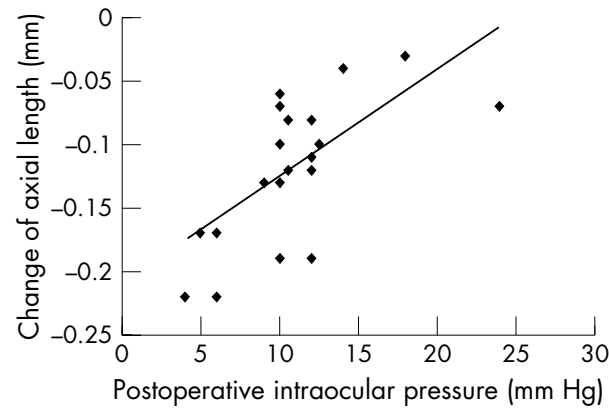

Figure 2 Correlation between change of axial length and postoperative intraocular pressure after combined operation $(p<0.002, y=0.008 x$ $-0.208, R^{2}=0.408$ )

alone $\left(p=0.264, R^{2}=0.095\right)$. Analysis of multivariates did not show a significant difference between the two treatment groups with regard to their correlation between the $\mathrm{AL}$ reduction and postoperative IOP $(p=0.20)$.

The mean surgical induced corneal astigmatism was +0.44 (0.83 dioptres - that is, WTR change (ranged from -1.28 dioptre to +1.85 dioptre) after combined operation. The surgical induced corneal astigmatism had no significant correlation with the interval between surgery and postoperative keratometry $(p=0.70)$. Thirteen $(54 \%)$ of the 24 eyes had WTR changes and 11 eyes (46\%) had ATR changes postoperatively. The mean induced astigmatism after the operation was 0.96 ( 0.54 dioptres at $90^{\circ}$ for the 13 eyes with WTR astigmatic changes, and 0.39 (0.38 dioptres at $180^{\circ}$ for the 11 eyes with ATR astigmatic changes. The WTR astigmatic change was significantly larger than the ATR astigmatic change $(\mathrm{p}<0.01)$.

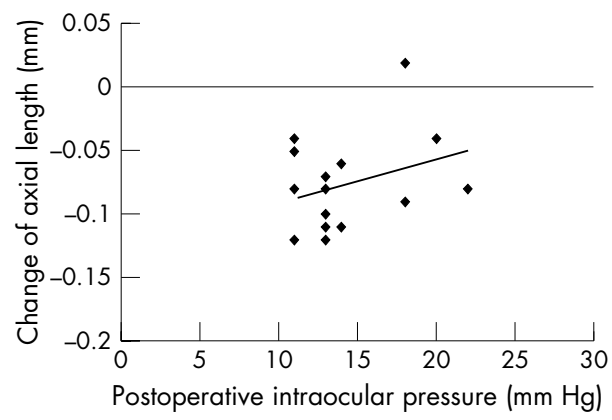

Figure 3 Correlation between change of axial length and postoperative intraocular pressure after cataract operation $(p=0.264, y=0.003 \times$ $-0.128, R^{2}=0.095$ ). 
Table 4 Comparisons between preoperative and postoperative values of cataract operation

\begin{tabular}{|c|c|c|c|c|}
\hline & Preoperative & Postoperative & $\begin{array}{l}\text { Difference between } \\
\text { preoperative and } \\
\text { postoperative } \\
\text { values }\end{array}$ & p Value \\
\hline Mean IOP $(\mathrm{mm} \mathrm{Hg})(\mathrm{SD})$ & $14.9(3.7)$ & $14.3(3.5)$ & $-0.5(3.7)$ & 0.58 \\
\hline Mean axial length $(\mathrm{mm})(\mathrm{SD})$ & $23.97(1.11)$ & $23.90(1.11)$ & $-0.08(0.04)$ & $<0.00001$ \\
\hline $\begin{array}{l}\text { Mean keratometry (dioptres) } \\
\text { (SD) }\end{array}$ & $43.65(1.43)$ & $43.77(1.48)$ & $0.12(0.28)$ & 0.13 \\
\hline $\begin{array}{l}\text { Mean refractive error } \\
\text { (spherical equivalent in } \\
\text { dioptres) (SD) }\end{array}$ & $-0.28(0.24)$ & $0.02(1.00)$ & $0.29(0.96)$ & 0.26 \\
\hline
\end{tabular}

\section{DISCUSSION}

The current study determined the AL with non-contact optical coherence biometry based on the principle of partial coherence interferometry. This method was reported to have a precision $(0.3-10 \mu \mathrm{m})$ and a resolution $(12 \mu \mathrm{m})$ more than 10 times greater than those of ultrasound..$^{14} 15$ The changes in AL after either the combined operation (117 (57) $\mu \mathrm{m})$ or the cataract operation alone (75 (38) $\mu \mathrm{m})$ were much greater than the precision and resolution of this biometric method. The IOLMaster applies two different fixed refractive indexes for determination of axial lengths of phakic and pseudophakic eye in the "Phakic" and "Pseudophakic Acryl" settings respectively. These two settings do not account for individual differences in the severity of cataractous lenses or the refractive property of IOL, and may introduce an artefact, which can bias the results. However, this potential bias was minimised by using the same method and same "Phakic" and "Pseudophakic Acryl" settings of measurement in both groups. another confounding factor was the effect of cataract surgery in altering the AL after a combined operation. The current study demonstrated that although cataract operation alone might be associated with an $\mathrm{AL}$ reduction, the reduction was significantly less than those after a combined operation. The baseline characteristics of the combined operation group did not differ significantly from the cataract operation group, except for a higher rate of diagnosis of glaucoma or glaucoma suspect in the combined operation group. This difference is expected because a higher rate of patients with coexisting cataract and glaucoma would benefit from a combined operation, but is unlikely to affect of the results of the current study.

Previous studies with contact ultrasound biometry demonstrated an AL reduction of $0.27 \mathrm{~mm}$ to $0.9 \mathrm{~mm}$ after trabeculectomy with or without mitomycin C. ${ }^{1-3}$ The mean reduction of 117 (57) $\mu \mathrm{m}$ reported in the current study after a combined operation is lower than those previously reported. Some possible explanations are the use of a more precise non-contact method of measurement, avoiding indentation or deformation of a soft globe after trabeculectomy, and absence of eyes with hypotony or hypotony related complication in the current study. Postoperative hypotony or related complications may result in a lower AL measured from oedema of the sclera, choroids, or retina; myopic refraction from forward displacement of the lens-iris diaphragm; or simply inconsistency in determination of the ocular dimensions and refraction. ${ }^{1}$

Although there are clear advantages of using non-contact optical coherence biometry to accurately determine the AL, it may not be applicable in eyes with advanced cataract. In enrolling only eyes with AL measurement by non-contact optical coherence biometry, this study might have eliminated the group of eyes with advanced cataract that had undergone combined operation, which might have a different amount of alteration of the ocular dimensions and surgical outcome.

Induction of a mean WTR corneal astigmatism after trabeculectomy with or without using mitomycin $\mathrm{C}$ had been reported..$^{3-8}$ However, studies on corneal astigmatism induction after a combined operation were few, and comparison among studies was limited by the different surgical techniques used. ${ }^{8}{ }^{9}$ Hong et al reported a shift of astigmatism at the vertical meridian from +2.17 dioptre to -1.72 dioptre over 12 months after a combined operation with the extracapsular cataract extraction technique that required a $10 \mathrm{~mm}$ corneoscleral incision and multiple sutures for wound closure. ${ }^{8}$ Another study on patients with combined operation with phacoemulsification technique reported a final WTR astigmatism of +0.49 dioptre. ${ }^{9}$ In that study, the corneoscleral incision was enlarged to $6.0 \mathrm{~mm}$ for inserting the nonfoldable IOL and mitomycin $\mathrm{C}$ was not used in all eyes. In the current study, a $3 \mathrm{~mm}$ temporal corneal incision was created for phacoemulsification, a single interrupted suture was used to close the corneal incision, and mitomycin $\mathrm{C}$ was used in all but one patient who had very thin conjunctiva. Although a tight corneal suture may have a countereffect on the WTR astigmatic induction from trabeculectomy, only one eye

Table 5 Comparison of changes of intraocular pressure (IOP), axial length, average keratometry, and refractive outcome between the combined operation group and cataract operation group

\begin{tabular}{llll}
\hline $\begin{array}{l}\text { Subtract preoperative values from } \\
\text { postoperative values }\end{array}$ & $\begin{array}{l}\text { Combined } \\
\text { operation group }\end{array}$ & $\begin{array}{l}\text { Cataract } \\
\text { operation group }\end{array}$ & p Value \\
\hline Mean IOP $(\mathrm{mm} \mathrm{Hg})(\mathrm{SD})$ & $-6.0(4.9)$ & $-0.5(3.7)$ & $<0.001$ \\
Mean axial length $(\mu \mathrm{m})(\mathrm{SD})$ & $-117(57)$ & $-75(38)$ & $<0.02$ \\
Mean keratometry (dioptres) (SD) & $0.23(0.46)$ & $0.12(0.28)$ & 0.39 \\
Mean refractive error (spherical & $0.22(0.91)$ & $0.29(0.96)$ & 0.80 \\
equivalent in dioptres) (SD) & & & \\
\hline
\end{tabular}


required corneal suture removal because of high corneal astigmatism induced in the current study. Tight scleral flap sutures in the presence of a posteriorly placed wound gape from internal sclerostomy were suggested to be the cause of induced WTR corneal astigmatism observed after trabeculectomy. ${ }^{16}$ Mitomycin $\mathrm{C}$ was shown to be associated with less induced WTR astigmatism in trabeculectomy, possibly related to the inhibitory effect of mitomycin $\mathrm{C}$ on wound healing. ${ }^{8}$ In addition, argon laser suture lysis of the scleral flap sutures may relieve the induced WTR corneal astigmatism. $66.7 \%$ of the eyes in the current study had at least one laser suture lysis performed postoperatively. Apparently, the induction of corneal astigmatism is largely dependent on the surgical technique. With the surgical technique used in the current study, there is an overall WTR corneal astigmatism induction.

Preoperative calculation of IOL power based on accurate measurements of the AL and the corneal curvature is crucial to attain desirable postoperative refraction. ${ }^{14}$ Although there was a significant reduction of AL after a combined operation and alteration of the corneal curvature, the current study did not find a significant difference between the predicted refraction and the refractive outcome in terms of spherical equivalent. The differences of the changes of average keratometry and refractive outcome between a cataract operation and a combined operation were not significant (table 5).

In summary, the current study demonstrates that with the use of a non-contact, precise biometric method, the AL is reduced significantly after a combined operation. In addition, the magnitude of AL reduction correlated significantly with the postoperative IOP. There was an overall WTR corneal astigmatic induction by this surgical technique. Despite the alteration of the AL and corneal curvature, the refractive outcome measured by spherical equivalent did not differ significantly from the predicted refraction. The finding of these relations may have clinical importance for choosing the surgical technique for patients who require a combined operation.

\section{Authors' affiliations}

S K Law, A M Mansury, D Vasudev, J Caprioli, Jules Stein Eye Institute, University of California, Los Angeles, CA, USA

Competing interests: none declared

Ethical approval: This study was approved by the institutional board of the University of California, Los Angeles.

\section{REFERENCES}

1 Nemeth J, Horoczi Z. Changes in the ocular dimensions after trabeculectomy. Int Ophthalmol 1992;16:355-7.

2 Cashwell LF, Martin CA. Axial length decrease accompanying successful glaucoma filtration surgery. Ophthalmology 1999;106:2307-11.

3 Kook MS, Kim HB, Lee SU. Short-term effect of mitomycin-C augmented trabeculectomy on axial length and corneal astigmatism. J Cataract Refract Surg 2001;27:518-23.

4 Hugkulstone CE. Changes in keratometry following trabeculectomy. $\mathrm{Br} J$ Ophthalmol 1991;75:217-18.

5 Rosen WJ, Mannis MJ, Brandt JD. The effect of trabeculectomy on corneal topography. Ophthalmic Surg 1992;23:395-8.

6 Claridge KG, Galbraith JK, Karmel V, et al. The effect of trabeculectomy on refraction, keratometry and corneal topography. Eye 1995;9(Pt 3):292-8.

7 Vernon SA, Zambarakii HJ, Potgieter F, et al. Topographic and keratometric astigmatism up to 1 year following small flap trabeculectomy (microtrabeculectomy). Br J Ophthalmol 1999;83:779-82.

8 Hong YJ, Choe CM, Lee YG, et al. The effect of mitomycin-C on postoperative corneal astigmatism in trabeculectomy and a triple procedure. Ophthalmic Surg Lasers 1998;29:484-9.

9 Gayton JL, Van der Karr MA, Sanders V. Combined cataract and glaucoma procedures using temporal cataract surgery. J Cataract Refract Surg 1996;22:1485-91.

10 Jampel HD, Friedman DS, Lubomski LH, et al. Effect of technique on intraocular pressure after combined cataract and glaucoma surgery: an evidence-based review. Ophthalmology 2002;109:2215-24.

11 Mamalis N. Intraocular lens power accuracy: how are we doing? J Cataract Refract Surg 2003;29:1-3.

12 Hitzenberger CK. Optical measurement of the axial eye length by laser Doppler interferometry. Invest Ophthalmol Vis Sci 1991;32:616-24.

13 Cravy TV. Calculation of the change in corneal astigmatism following cataract extraction. Ophthalmic Surg 1979;10:38-49.

14 Drexler W, Findl O, Menapace R, et al. Partial coherence interferometry: a novel approach to biometry in cataract surgery. Am J Ophthalmol 1998;126:524-34.

15 Findl O, Drexler W, Menapace R, et al. High precision biometry of pseudophakic eyes using partial coherence interferometry. J Cataract Refract Surg 1998;24:1087-93.

16 The Fluorouracil Filtering Surgery Study Group. Risk factors for suprachoroidal hemorrhage after filtering surgery. Am J Ophthalmol 1992;113:501-7. 